\title{
OBTENÇÃO DE NANOWHISKERS A PARTIR DE FIBRAS DE BAMBU PARA USO EM NANOCOMPÓSITOS*
}

\author{
Kleiton dos Santos Melo ${ }^{1}$ \\ Francisco Marcellus Carmo do Nascimento ${ }^{1}$ \\ Santino Loruan Silvestre de Melo ${ }^{2}$ \\ Milene Muniz Eloy da Costa ${ }^{3}$ \\ Enio Pontes de Deus ${ }^{4}$
}

\section{Resumo}

As fibras de bambu são ricas em celulose, hemicelulose e lignina. Elas apresentam altos módulos de elasticidade e alta resistência mecânica, o que as tornam bastante eficazes como reforço em compósitos. Com o intuito de aprimorar as propriedades mecânicas de materiais poliméricos em escalas diminutas, tem-se buscado a aplicação desses reforços em sua forma nano. A extração de nanocelulose tem sido bastante estudada e, em nosso trabalho, foram feitas adaptações de métodos que mostraram-se mais eficazes. Os nanowhiskers a base de bambu foram obtidos por hidrólise ácida a $64 \%$, após tratamento químico prévio de limpeza e branqueamento. Os materiais obtidos foram então caracterizados por FTIR, TG, DTG e tamanho de partícula por Potencial Zeta, onde seus resultados indicaram que o método de produção utilizado foi eficaz.

Palavras-chave: Bambu; Nanowhiskers; Nanopartículas de celulose; Nanocompósitos.

\section{OBTAINING OF NANOWHISKERS FROM BAMBOO FIBERS FOR USE IN NANOCOMPOSITES}

\section{Abstract}

Bamboo fibers are rich in cellulose, hemicellulose and lignin. They present high elastic modulus and high mechanical strength, which makes them very effective as reinforcement in composites. In order to improve the mechanical properties of polymeric materials in very small scales, the application of these reinforcements in their nano form has been investigated. The nanocellulose extraction has been studied a lot and, in our work, adjustments in some methods that showed to be more effective were done. The nanowhiskers made from bamboo was obtained by $64 \%$ of acid hydrolisis after prior chemical treatment of cleaning and bleaching. The materials were then characterized by FTIR, TG, DTG and particle size by Zeta Pontential, which indicated that the production method was effective.

Keywords: Recycled polypropylene; Bamboo fibers; Polymeric composites; Surface Modifiers.

1 Engenharia Metalúrgica, graduando, Iniciação Científica, Departamento de Engenharia Metalúrgica e de Materiais, Universidade Federal do Ceará, Fortaleza, Ceará, Brasil.

2 Bacharel em Física, pós-graduando, Mestrado, Departamento de Engenharia Metalúrgica e de Materiais, Universidade Federal do Ceará, Fortaleza, Ceará, Brasil.

3 Engenheira Química, mestre em Engenharia Industrial, pós-graduanda, Doutorado, Departamento de Engenharia Metalúrgica e de Materiais, Universidade Federal do Ceará, Fortaleza, Ceará, Brasil.

4 Engenheiro Civil, doutor em Engenharia Estrutural, professor/orientador, Departamento de Engenharia Metalúrgica e de Materiais, Universidade Federal do Ceará, Fortaleza, Ceará, Brasil. 


\section{INTRODUÇÃO}

As fibras naturais vegetais destacam-se por serem abundantes e por consumirem reduzida quantidade de energia em sua produção, quando comparado ao consumo necessário para a fabricação das fibras sintéticas. Estas fibras, também chamadas de fibras lignocelulósicas, podem ser definidas como compósitos naturais, pois apresentam uma matriz de lignina reforçada com celulose [1].

Dentre as fibras lignocelulósicas, destacam-se as de bambu, pois tratam-se de materiais amplamente utilizados como reforços em compósitos poliméricos, devido, principalmente, às suas propriedades mecânicas, possibilitando, assim, incremento da eficiência mecânica da matriz. Além disso, trata-se de um vegetal de fácil reflorestamento (sustentável e inesgotável), com uso de pequena área de plantio por alta quantidade de material e, por não ser amplamente empregado na indústria, não apresenta competição de mercado [2].

Diminuindo consideravelmente a escala, os materiais lignocelulósicos podem ser utilizados também como reforço de nanocompósitos poliméricos. Estes consistem em compósitos reforçados com materiais em escala nanométrica [2]. Normalmente, as nanopartículas utilizadas nesses compósitos são nanofibras de celulose [3]. Estas podem incrementar a resistência mecânica do material, sem interferir no seu índice de alongamento, e também o seu módulo de elasticidade. Essas mudanças são consequência da maior área superficial apresentada pelas nanofibras, além da atenuação de possíveis defeitos formados durante o preparo dos compósitos. Em somatório, fibras com diâmetros inferiores a $100 \mathrm{~nm}$ podem ser utilizadas no desenvolvimento de nanocompósitos transparentes, pois, por tratar-se de dimensões nanométricas, não afetam a transparência dos polímeros [2].

As nanofibras de celulose, ou nanowhiskers, podem ser obtidas a partir de diferentes fontes de fibras naturais, inclusive vegetais, como algodão, cana-de-açúcar, bambu, etc. O que torna o Brasil um potencial produtor, visto sua imensa variedade florestal. As propriedades e dimensões desses materiais dependem, basicamente, da origem da celulose e do processo adotado para sua produção [4].

Nanowhiskers de celulose (CNW) podem ser preparados por hidrólise ácida em uma gama de materiais lignocelulósicos [5]. A hidrólise ácida com ácido sulfúrico $\left(\mathrm{H}_{2} \mathrm{SO}_{4}\right)$ $64 \%$ tem se mostrado como o método mais simples e comum para obtenção dos nanowhiskers, pois promove uma rápida incorporação e destruição das cadeias de hemicelulose, que, consequentemente, destroem as microfibrilas, restando apenas pequenos cristais de celulose, a própria nanocelulose $[4,6]$.

De maneira semelhante ao uso das fibras longas, os nanowhiskers provenientes do bambu, e de qualquer fonte vegetal, apresentam a desvantagem de possuírem baixa adesão com a matriz polimérica, o que pode fragilizar o nanocompósito final [7]. Assim, outros tratamentos prévios de limpeza e branqueamento também são necessários, com o intuito de retirar alguns constituintes das fibras e facilitar sua incorporação em outros materiais [6].

Com o objetivo de produzir nanowhiskers de celulose a partir de fibras de bambu com possível aplicação como reforço em compósitos poliméricos, foram avaliados o método de obtenção dos nanowhiskers, bem como o efeito dos tratamentos e, em somatório, foi realizado uma análise de rendimento de material, comparando a quantidade utilizada no início e a quantidade residual. 


\section{MATERIAIS E MÉTODOS}

\subsection{Obtenção de nanopartículas de bambu}

As fibras de bambu foram obtidas de touceiras presentes na Universidade Federal do Ceará (UFC). Seus colmos foram usinados em serra fita automática na Oficina do Departamento de Física, no Centro de Ciências no campus do Pici da UFC, para separação dos nós e entrenós. A região escolhida para retirada do material fibroso foi a dos entrenós próximos a casca, pois trata-se do local com maior quantidade de fibras, consequentemente com maior grau de celulose.

A partir da região escolhida, os entrenós tiveram suas paredes laminadas longitudinalmente de maneira manual no Laboratório de Mecânica da Fratura e Fadiga (LAMEFF) da UFC. Em seguida, foram realizados cortes finos em seções longitudinais para a realização do desfibramento, também manual.

Com as fibras retiradas, elas foram micronizadas em trituradora a vácuo e posteriormente separadas em peneiras de 60 mesh. O material utilizado nas próximas etapas foram as nanopartículas retidas na peneira.

\subsection{Tratamento químico: Limpeza e Branqueamento}

Após o processo de desfibramento e de micronização, as nanopartículas passaram por algumas reações a fim de limpar e branquear suas superfícies mais propícias a adesão com a matriz polimérica. O tratamento das nanopartículas consistiu em mercerização e branqueamento.

\subsubsection{Mercerização}

O processo tem o objetivo de remover ceras, lignina e açúcares. A mercerização age na superfície do material, expondo sua estrutura fibrilar interna, o que promove o aumento de sua área superficial e, consequentemente, o aumento da rugosidade da superfície, que favorece sua ancoragem na matriz.

As nanopartículas foram imersas em solução aquosa $2 \%(\mathrm{~m} / \mathrm{v})$ de hidróxido de sódio $(\mathrm{NaOH})$ e aquecidas à $80^{\circ} \mathrm{C}$ por 4 horas sob agitação magnética. Posteriormente, foram lavadas e separadas.

O procedimento de imersão foi repetido durante 6 vezes, até o momento em que foi observado a manutenção da coloração da solução na presença das nanopartículas. Após essa etapa, elas foram acondicionadas em estufa à $60^{\circ} \mathrm{C}$ por 24 horas.

\subsubsection{Branqueamento}

O processo tem o objetivo de promover, como o próprio nome diz, o branqueamento, ou clareamento, das nanopartículas.

As nanopartículas mercerizadas e secas foram imersas em solução aquosa $2 \%(\mathrm{v} / \mathrm{v})$ de hipoclorito de sódio $(\mathrm{NaClO})$ e aquecidas à $80{ }^{\circ} \mathrm{C}$ por 6 horas sob agitação mecânica. Posteriormente, foram lavadas e separadas, resultando em uma polpa celulósica.

O procedimento completo foi repetido 3 vezes, até que as nanopartículas apresentassem um elevado grau de alvura. Após essa etapa, a polpa resultante foi acondicionada em estufa à $60^{\circ} \mathrm{C}$ por 24 horas. 


\subsection{Tratamento químico: Hidrólise ácida}

Findados os tratamentos anteriores, a polpa de celulose seca seguiu para a etapa de obtenção da nanocelulose. Esse processo consistiu na imersão do material resultante em uma solução aquosa $64 \%(\mathrm{v} / \mathrm{v})$ de ácido sulfúrico $\left(\mathrm{H}_{2} \mathrm{SO}_{4}\right)$. A mistura foi acondicionada em balão volumétrico, mantido sob agitação magnética e aquecimento de $55^{\circ} \mathrm{C}$ por $15 \mathrm{~min}$. Foram utilizados $12 \mathrm{~mL}$ de solução para cada grama de polpa de celulose seca.

Após o tratamento, a polpa resultante seguiu para o processo de filtragem em bomba de vácuo com filtro de papel. O material resultante, a nanocelulose, foi levada para banho de ultrassom por $40 \mathrm{~min}$, com o intuito de dispersar as partículas imersas em água destilada. O material foi então levado para a centrifugação durante 30 minutos a 4000 RPM até que o sobrenadante fosse acondicionado no fundo. O processo do banho e da centrifugação foram repetidas até pH neutro.

\section{RESULTADOS E DISCUSSÃO}

As amostras foram caracterizadas quimicamente com a técnica de infravermelho com transformada de Fourier (FTIR), termicamente com a análise de termogravimetria (TG) e de termogravimetria derivada (DTG) e espectroscopicamente com o ensaio de Potencial Zeta, que analisa a distribuição de comprimento das partículas.

Os infravermelhos foram obtidos em um equipamento da marca Perkin Elmer, modelo Ezimer 200, na região de $400-4000 \mathrm{~cm}^{-1}$. As amostras de nanowhiskers foram previamente secas, misturadas e analisadas em pastilhas de $\mathrm{KBr}$. Uma diferença significativa entre os espectros das fibras é a presença de bandas com intensidade elevada em $1730 \mathrm{~cm}-1$ e $1050 \mathrm{~cm}-1$, que caracteriza o aparecimento da banda C-O. As intensidades dos picos que caracterizam os estiramentos provenientes da celulose evidenciaram que os processos de limpeza e hidrólise da fibra foram eficientes. Abaixo a Figura 1 apresenta o infravermelho dos nanowhiskers obtidos.

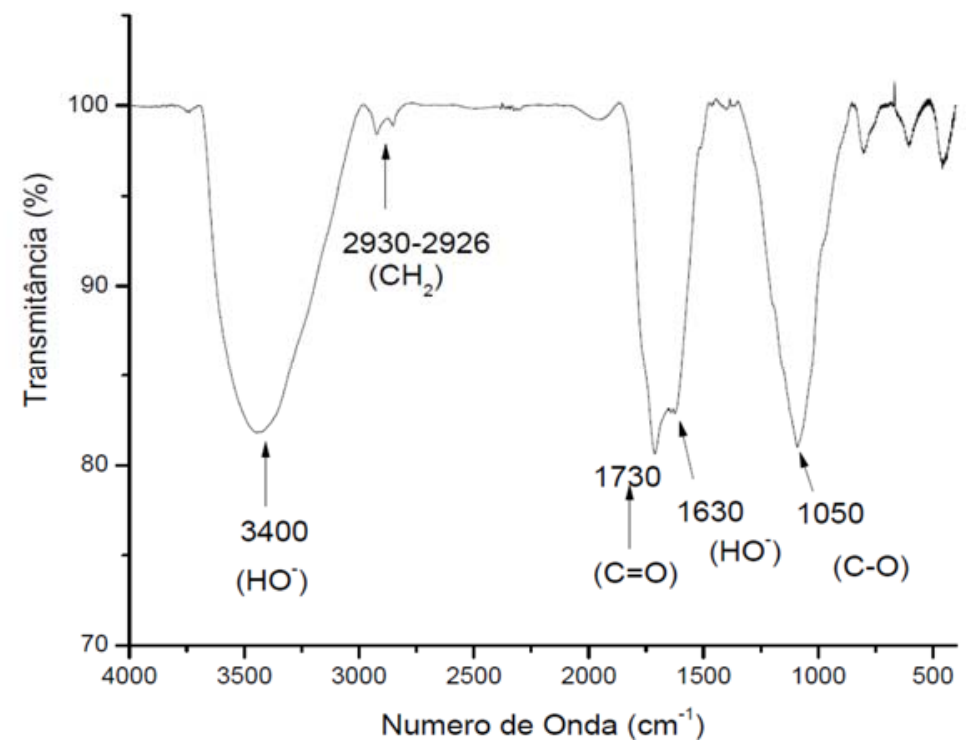

Figura 1. FTIR dos nanowhiskers obtidos.

A Tabela 1 abaixo apresenta os comprimentos de onda mais importantes para a compreensão da análise de infravermelho da amostra. Com esses resultados, podese garantir que ocorreu remoção de parte da lignina e hemicelulose, com diminuição 
das intensidades de determinadas bandas em comparação com imagens de infravermelho de fibras de bambu de trabalhos anteriores.

Tabela 1. Bandas de absorção de FTIR referente aos nanowhiskers produzidos.

\begin{tabular}{|c|c|}
\hline Comprimento de onda $\left(\mathrm{cm}^{-1}\right)$ & Bandas de absorção \\
\hline 3450 & Estiramento $\mathrm{O}-\mathrm{H}$ \\
\hline 2930 & Estiramento $\mathrm{C}-\mathrm{H}$ \\
\hline 1630 & Deformação $\mathrm{O}-\mathrm{H}$ da Água \\
\hline 890 & Estiramento Aromático, $\mathrm{C}-\mathrm{H}$ Deformação \\
\hline
\end{tabular}

A Figura 2 apresenta o comportamento térmico da nanocelulose. É perceptível a presença de dois eventos térmicos, um em $54{ }^{\circ} \mathrm{C}$ que pode ser atribuído a perca de água e em $330^{\circ} \mathrm{C}$ que indica a degradação da celulose. Também é notória no gráfico abaixo, que a degradação continua a altas temperaturas $\left(600^{\circ} \mathrm{C}\right)$, podendo ser observado uma perda de $55 \%$ da massa total do material.

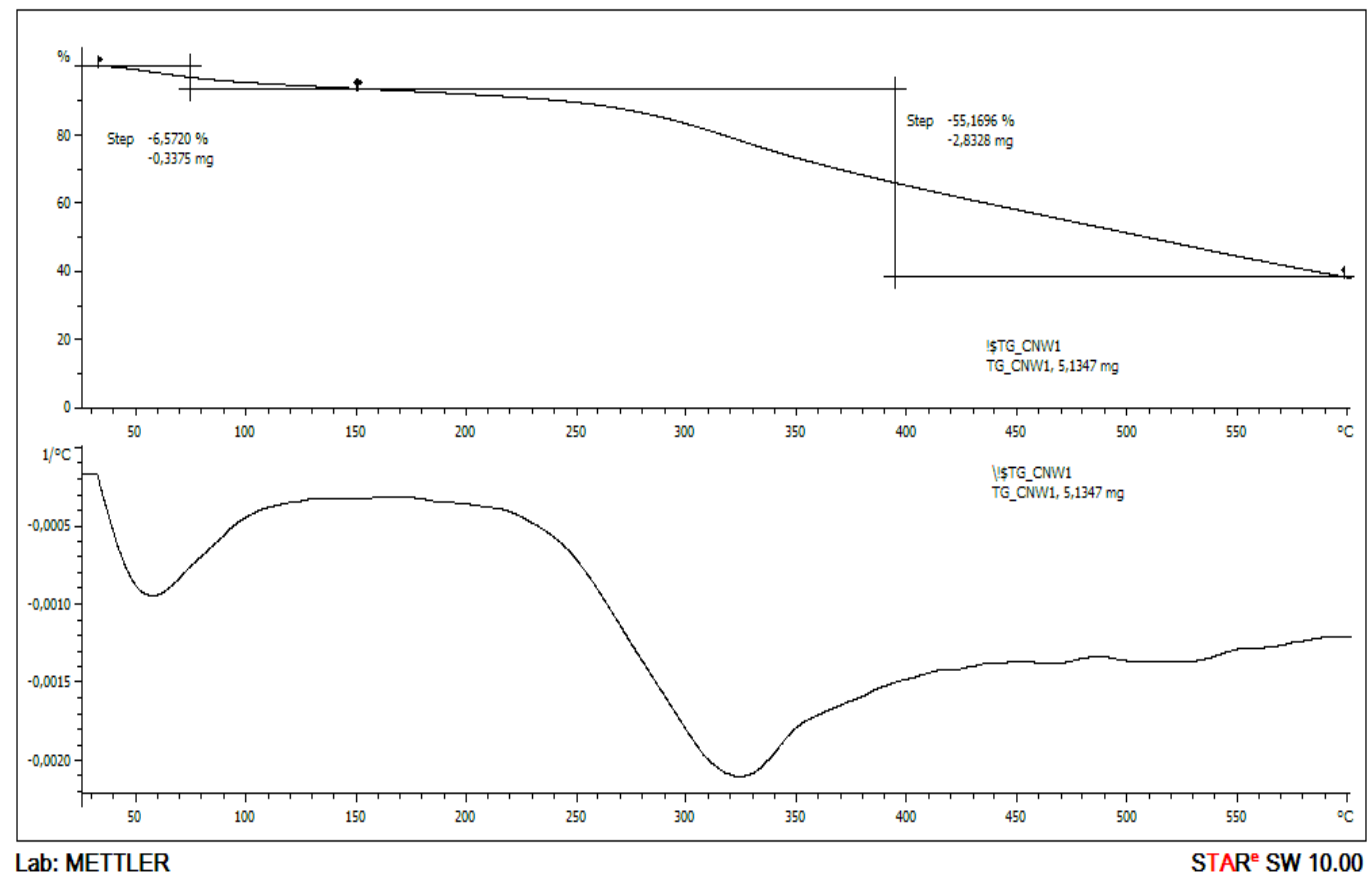

Figura 2. TG e DTG dos nanowhiskers obtidos.

O gráfico de Potencial Zeta de partículas é utilizado na avaliação de forças eletrostáticas repulsivas, avaliando, dentre outros parâmetros, a estabilidade da suspensão de partículas. Observando a Figura 3, percebe-se que $30 \%$ das partículas obtiveram comprimentos abaixo de $100 \mathrm{~nm}$, sendo associado ao diâmetro das nanopartículas. Enquanto o restante obteve comprimento entre $100 \mathrm{~nm}$ até $800 \mathrm{~nm}$, que foram associamos aos whiskers de celulose. 


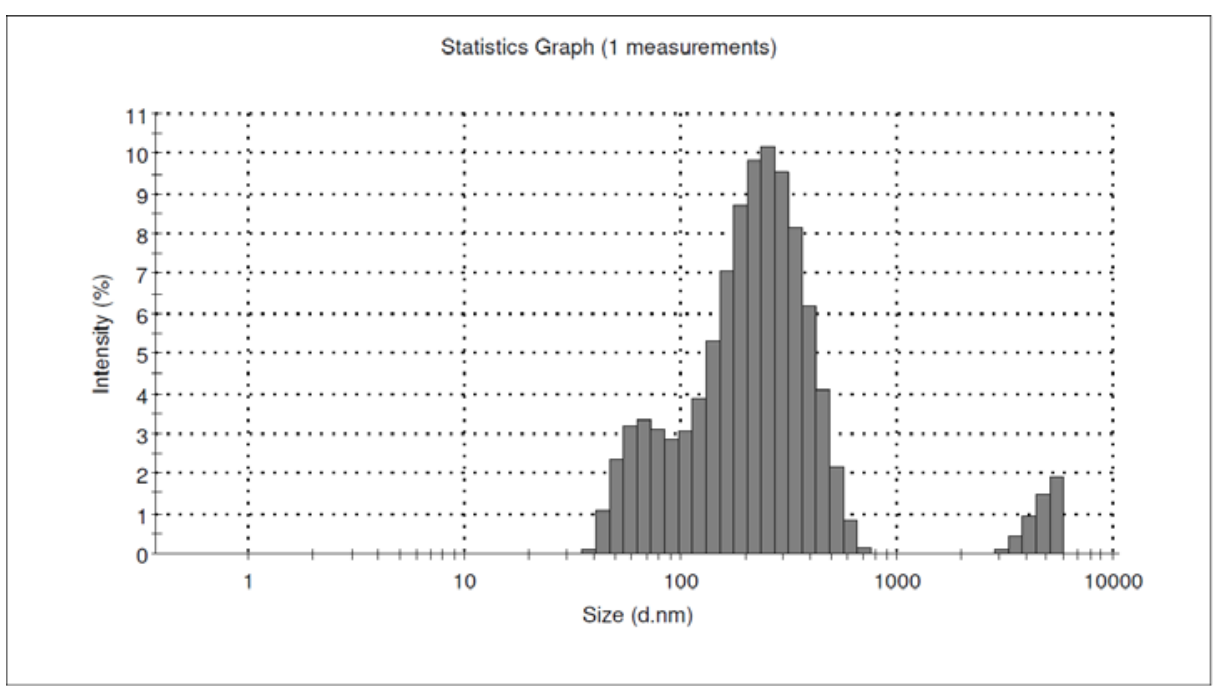

Figura 3. Potencial Zeta dos nanowhiskers obtidos.

\section{CONCLUSÃO}

Após todo o processo de obtenção dos nanowhiskers, pode-se afirmar que o método empregado foi eficiente, pois as caracterizações utilizadas apresentaram fortes indícios de aparecimento dessas nanopartículas.

Sugere-se como próxima etapa, avaliar a adesão desses materiais na matriz polimérica, evidenciando se os tratamentos realizados foram suficientes para promover a compatibilização.

\section{Agradecimentos}

Os autores são gratos à Coordenação de Aperfeiçoamento de Pessoal de Nível Superior (CAPES) pelo apoio financeiro.

\section{REFERÊNCIAS}

1 Satyanarayana KG, Sukumaran K, Mukherjee OS, Pavithron C, Pillai SGK. Polymer Composites Ciment \& Concrete Composites. 1990; 12: 117-136.

2 Guimarães Jr M, Novack KM, Botaro VR. Caracterização anatômica da fibra de bamboo (Bambusa vulgaris) visando sua utilização em compósitos poliméricos. Revista Iberoamericana de Polímeros. 2010; 11: 442-456.

3 Mandal A, Chakrabarty D. Studies on the mechanical, thermal, morphological and barrier properties of nanocomposites based on poly(vinyl alcohol) and nanocellulose from sugarcane bagasse. Journal of Industrial and Engineering Chemistry. 2014; 20 : 462-473.

4 Silva JBA, Miranda NM, Vargas JFP, Druzian JI. Preparação de nanowhiskers de celulose a partir do resíduo da fibra de piaçava da palmeira Attalea funifera martius. $19^{\circ}$ Congresso Brasileiro de Engenharia e Ciência dos Materiais - CBECiMat. 2010: 36623669.

5 Haafiz MKM, Hassan A, Zakaria Z, Inuwa IM. Isolation and characterization of cellulose nanowhiskers from oil palm biomass microcrystalline cellulose. Carbohydrate Polymers. 2014; 103(15): 119-125.

6 Cherian BM, Pothan LA, Nguyen-Chuen T, Menning G, Kottaisamy M, Thomas S. A novel method for the synthesis of cellulose nanofibril whiskers from banana fibers and characterization. Journal of Agricultural and Food Chemistry. 2008; 56: 5617-5627.

7 Hopewell J, Dvorak R, Kosior E. Plastic recycling: challenges and opportunities. Philosophical Transactions of the Royal Society B: Biological Science. 2009; 364(1526): 2115-2126. 